\title{
\begin{tabular}{l|l} 
MitTraries & DSpace@MIT
\end{tabular}
}

\author{
MIT Open Access Articles
}

\section{Morphology and processing of aligned carbon nanotube carbon matrix nanocomposites}

The MIT Faculty has made this article openly available. Please share how this access benefits you. Your story matters.

Citation: Stein, Itai Y., and Brian L. Wardle. “Morphology and Processing of Aligned Carbon Nanotube Carbon Matrix Nanocomposites." Carbon 68 (2014): 807-813.

As Published: http://dx.doi.org/10.1016/j.carbon.2013.12.001

Publisher: Elsevier

Persistent URL: http://hdl.handle.net/1721.1/110454

Version: Author's final manuscript: final author's manuscript post peer review, without publisher's formatting or copy editing

Terms of use: Creative Commons Attribution-NonCommercial-NoDerivs License 


\title{
Morphology and Processing of Aligned Carbon Nanotube Carbon Matrix Nanocomposites
}

\author{
Itai Y. Stein* \\ Department of Mechanical Engineering, Massachusetts Institute of Technology, \\ 77 Massachusetts Ave, Cambridge, MA 02139, USA. \\ Brian L. Wardle ${ }^{\dagger}$ \\ Department of Aeronautics and Astronautics, Massachusetts Institute of Technology, \\ 77 Massachusetts Ave, Cambridge, MA 02139, USA.
}

\begin{abstract}
Intrinsic and scale-dependent properties of carbon nanotubes (CNTs) have led aligned CNT architectures to emerge as promising candidates for next-generation multifunctional applications. Enhanced operating regimes motivate the study of CNT-based aligned nanofiber carbon matrix nanocomposites (CNT A-CMNCs). However, in order to tailor the material properties of CNT A-CMNCs, porosity control of the carbon matrix is required. Such control is usually achieved via multiple liquid precursor infusions and pyrolyzations. Here we report a model that allows the quantitative prediction of the CNT A-CMNC density and matrix porosity as a function of number of processing steps. The experimental results indicate that the matrix porosity of A-CMNCs comprised of $\sim 1 \%$ aligned CNTs decreased from $\sim 61 \%$ to $\sim 55 \%$ after a second polymer infusion and pyrolyzation. The model predicts that diminishing returns for porosity reduction will occur after 4 processing steps (matrix porosity of $\sim 51 \%$ ), and that $>10$ processing steps are required for matrix porosity $<50 \%$. Using this model, prediction of the processing necessary for the fabrication of liquid precursor derived A-CMNC architectures, with possible application to other nanowire/nanofiber systems, is enabled for a variety of high value applications.
\end{abstract}

\section{INTRODUCTION}

The tailorable energy harvesting ${ }^{1-8}$, storage $^{7-11}$, and dissipation ${ }^{12-16}$ capabilities of nanowires, nanofibers, and nanotubes make them prime candidates for next generation multifunctional material architectures. By integrating aligned nanofibers with a carbon matrix, materials with high strength, toughness, low density, and extended operating regimes may be synthesized. These materials can be produced through the heat treatment (pyrolysis) of aligned nanofiber polymer matrix nanocomposite (A-PNC) precursors, which are analogous to the APNCs reported elsewhere ${ }^{15-24}$. Such processing follows the typical synthesis routes of polymer derived ceram$\mathrm{ics}^{25-28}$, but relies on capillary ${ }^{15-21}$ or vacuum ${ }^{21-24}$ assisted wetting of the aligned nanofibers rather than surfactant or functionalization assisted mixing. In order to manufacture aligned nanofiber carbon matrix nanocomposites (A-CMNCs) with tailored properties, the porosity of the matrix, which strongly influences its material properties, must be controlled. Such control over matrix porosity can be achieved via multiple polymer infusions and subsequent pyrolyzations. But since a theoretical model that quantifies the porosity change as a function of processing currently does not exist, there is no way to predict whether an A-CMNC with a certain matrix porosity can be fabricated, or how many processing steps would be necessary to achieve the desired matrix morphology. Here we report a model that allows the quantitative prediction of the A-CMNC density and matrix porosity as a function of number of polymer infusions and pyrolyzations. We find that diminishing returns will be reached after the fourth polymer infusion and pyrolyzation, and that the common wet infusion processing method may not be a viable approach for making low porosity $(<20 \%)$ A-CMNCs.

To evaluate the performance of the reinfusion model, application to an exemplary system of nanofibers was necessary. This system consisted of $\sim 8 \mathrm{~nm}$ outer diameter aligned multiwalled carbon nanotubes (CNTs), which have interesting intrinsic mechanical ${ }^{29-33}$ and transport $^{33-39}$ properties, and were previously used to fabricate A-CMNCs with a pyrolytic carbon $(\mathrm{PyC})$ matrix via the pyrolysis of phenolic matrix A-PNC precursors ${ }^{23,24}$. See Fig. 1 for an illustration of a CNT A-CMNC architecture after a single polymer infusion and pyrolyzation (Fig. 1a), and after a double polymer infusion and pyrolyzation (Fig. 1b) illustrating a decrease in the $\mathrm{PyC}$ matrix porosity from $\sim 50 \%$ (Fig. 1a) to $\sim 25 \%$ (Fig. 1b). Previous studies on the morphology of the CNT arrays, known as forests, indicate that the average inter-CNT spacing in the as-grown ( 1 vol. \%) CNT forests is $\sim 80 \mathrm{~nm}^{18,40}$, meaning that the fabrication of low porosity A-PNCs is possible. However, once the polymer precursors are heat treated, additional porosity forms, and modeling indicates that its reduction to a value similar to that of carbon fiber reinforced carbon matrix composites $(\lesssim 20 \%)^{28}$ may not be possible using wet infusion in A-CMNCs. The primary goal of this study is to develop a framework that allows the prediction of the attainable matrix porosity as a function of number of polymer infusions in A-CMNCs for infusions performed using a liquid precursor with varying degrees of dilution. By developing and applying this reinfusion model to aligned nanowire and nanofiber systems, better prediction of the attainable matrix morphology using wet polymer infu- 
(a) One Infusion and Pyrolyzation

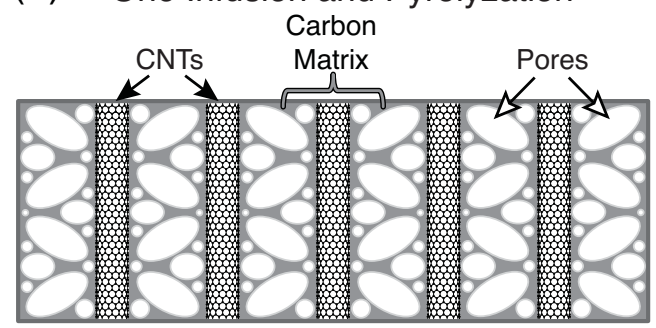

(b) Two Infusions and Pyrolyzations

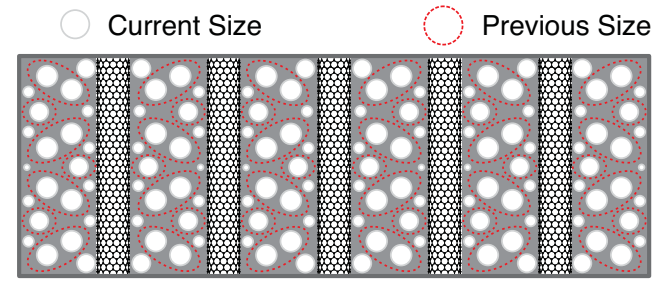

Aligned-CNT Carbon Matrix Nanocomposite

FIG. 1. Illustration of the structure of a CNT A-CMNC after a single polymer infusion and pyrolyzation (a) and after a double polymer infusion and pyrolyzation (b). The figure illustrates a reduction of PyC matrix porosity from $\sim 50 \%$ (a) to $\sim 25 \%$ (b).

sion can be achieved, which could lead to macroscopic material architectures with optimized properties. Using this knowledge, multifunctional material solutions for aerospace, structural, and power system applications can potentially be designed and manufactured.

\section{MODEL DEVELOPMENT}

\section{A. A-CMNC Density as a Function of Polymer Infusions}

The A-CMNC porosity after a single polymer infusion, $\phi_{m, 1}$, can be defined as a function of the initial CNT array porosity, $\phi_{o}$, density of the polymer in the pores, $\rho_{\text {pnc, } 1}$, the density of the ideal graphitic matrix, $\rho_{g}\left(\rho_{g} \simeq\right.$ $2.25 \mathrm{~g} / \mathrm{cm}^{3}$, see Section S2 Supplementary Materials for details), the volume change coefficient (due to polymer infusion), $\Delta V_{\text {inf }}$, the volume change coefficient (due to pyrolysis), $\Delta V_{p, 1}$, and mass change coefficient (due to pyrolysis), $\Delta M_{p, 1}$ :

$$
\begin{array}{r}
\phi_{m, 1}=\left(1-\frac{1-\phi_{o}}{\left(1+\Delta V_{i n f}\right)\left(1+\Delta V_{p, 1}\right)}\right) \\
\left(1-\frac{\rho_{p n c, 1}\left(1+\Delta M_{p, 1}\right)}{\rho_{g}\left(1+\Delta V_{p, 1}\right)}\right)
\end{array}
$$

Because no change in CNT array volume was observed post infusion $\left(\Delta V_{\text {inf }} \simeq 0\right)$, the $\Delta V_{\text {inf }}$ term was omitted from the remaining equations presented here, but it can be re-introduced into Eq. 3 if necessary for nanofiber arrays with lower $\phi_{o}$ values (volume fractions $>1 \%$ ), such as densified CNT arrays ${ }^{15,16,18,24,40}$. Because Eq. 1 is more instructive when expressed in terms of density, it was multiplied by a factor of $\rho_{g}$, leading to the definition of the matrix porosity after the first infusion, $\rho_{m, 1}$ (see Eq. S1 in the Supplementary Materials). For subsequent infusions, the increase in density may vary (i.e. slow down), so $\rho_{m, 1}$ needs to be modified to account for a non-constant rate of change by using the new mass and volume of the system. See Eq. S2-S3 in the Supplementary Materials for derivation of the working equation that is valid for an $i$ number of phenolic infusions and pyrolyzations (Eq. S3). To condense Eq. S3 from the Supplementary Materials, three additional terms need to be used: the unit step function $(H(x))$, the amount of porosity filled by each infusion $\left(\xi_{i}\right)$, and a filling coefficient accounting for the difficulty of filling the pores $(\psi)$. $\psi$ physically represents the increasingly diminishing returns of each successive infusion and pyrolyzation. $H(x)$, $\xi_{i}, \psi$ are defined as follows:

$$
\begin{gathered}
H(x)= \begin{cases}0 & x<0 \\
1 & x \geq 0\end{cases} \\
\xi_{i}=\frac{\rho_{p n c, i}\left(1+\Delta M_{p, i}\right)}{\rho_{g}\left(1+\Delta V_{p, i}\right)} \\
\xi_{i}= \begin{cases}\xi_{i} & i \leq 2 \\
\psi^{i-2} \xi_{2} & i>2\end{cases}
\end{gathered}
$$

Using Eq. 2a-c in conjunction with Eq. S3 from the Supplementary Materials leads to the following general form of $\rho_{m, i}$ :

$$
\begin{array}{r}
\rho_{m, i}=\rho_{g}\left(1-\frac{1-\phi_{o}}{1+\Delta V_{p}}\right)\left(1-\left(\xi_{1}\right)(H(i)\right. \\
+\left(1-\xi_{2}\right)(H(i-2) \\
-\left(\sum_{j=3}^{i} \psi^{j-2} \xi_{2}\left(1-\psi \xi_{2} H(i-4)\right)\right. \\
\\
\left.\left.\left.\left.\left(1-\sum_{k=5}^{i} \prod_{h=5}^{k} \psi^{h-4} \xi_{2}\right)\right)\right)\right)\right)
\end{array}
$$

Since the volume is unlikely to change after the first pyrolyzation when carbonization takes place at relatively low temperatures $\left(\lesssim 1000^{\circ} \mathrm{C}\right)$ and atmospheric pressure, the following simplification was made in Eq. 3: $\Delta V_{p, 1} \equiv \Delta V_{p}$ and $\Delta V_{p, k \neq 1} \simeq 0$. As discussed in Section IV, such a simplification cannot be made when high pressures are used to densify the $\mathrm{PyC}$ matrix during py$\operatorname{rolysis}^{28}\left(\Delta V_{p, k>1} \not 0\right)$.

To obtain the density of the A-CMNCs as a function of $i$, the contribution of the CNTs, represented by their 
average intrinsic density $\left(\rho_{c n t}\right)$, needs to added to $\rho_{m, i}$ yielding the following form:

$$
\rho_{c, i}=\rho_{m, i}+\rho_{c n t}\left(\frac{1-\phi_{o}}{1+\Delta V_{p}}\right)
$$

However, before Eq. 4 can be used, $\rho_{c n t}$ must be determined for the specific CNTs that comprise the ACMNC. Using the average values of the multilwalled CNT (MWCNT) inner, $D_{i}$, and outer, $D_{o}$, diameters from a previous high resolution transmission electron microscopy (HRTEM) study ${ }^{41}$, and the relationship derived for $\rho_{c n t}$ as a function of their number of CNT walls, $\rho_{c n t}$ is evaluated at $\sim 1.7 \mathrm{~g} / \mathrm{cm}^{3}$ (see Section S2 in the Supplementary Materials for details), which is in very good agreement with the values predicted by a previously reported model for the intrinsic density of $\mathrm{CNTs}^{42}$.

Using Eq. 4 , the evaluated $\rho_{c n t}$, and the experimentally determined CNT A-CMNC density $\left(\rho_{c, i}^{\exp }\right)$, mass $\left(\Delta M_{p, i}^{e x p}\right)$ and volume change $\left(\Delta V_{p, i}^{e x p}\right)$ coefficients, the experimental matrix porosity $\left(\phi_{m, i}^{e x p}\right)$ can be calculated, and predictions after $i$ infusions can be quickly and easily generated. Also, this model can be used quantify the processing of carbon matrix composites comprised of other nanowire/nanofiber arrays, or hierarchical systems where the nanowires/nanofibers are grown on micron-scale fibers, such as fuzzy fiber composites ${ }^{43,44}$, as long as the intrinsic mass of the micron-scale fibers and nanowires/nanofibers is either known or can be determined (either empirically or theoretically). Using these results, the point of diminishing infusion returns and the maximum attainable density of A-CMNCs for a variety of other nanowire and nanofiber systems can be predicted without performing the very time consuming full parametric study that would be necessary for their empirical determination.

\section{EXPERIMENTAL}

Here we describe the methodology used to investigate the density of CNT-based A-CMNCs comprised of $\sim 1$ vol. $\%, \sim 8 \mathrm{~nm}$ outer diameter, vertically aligned MWCNTs.

\section{A. CNT A-CMNC Fabrication Methods}

Aligned CNT arrays were grown via a previously described thermal catalytic chemical vapor deposition process using ethylene as the carbon source ${ }^{15-18}$. The forests were grown on $1 \mathrm{~cm} \times 1 \mathrm{~cm}$ Si substrates to form CNT arrays that are $\sim 1 \mathrm{~mm}$ tall, have an average inter-CNT spacing of $\sim 80 \mathrm{~nm}^{40}$, and volume fractions of $\sim 1 \%$ $\mathrm{CNTs}^{41}$. A post-growth $\mathrm{H}_{2}$ anneal ${ }^{45}$ is used to weaken the attachment of the CNTs to the catalyst layer, which enables the easy delamination of the CNT forest from the Si substrate using a standard lab razor blade, thereby allowing further CNT processing to be performed in their free-standing state. See Fig. 2a for a high resolution scanning electron microscopy (HRSEM) micrograph of an asgrown CNT forest.

Fabrication of CNT A-PNCs via vacuum assisted wetting was performed by first gently depositing freestanding CNT forests into hollow cylindrical plastic molds, ensuring that the primary axis of the CNTs in the forest was orthogonal to the plane of the mold. The CNT forest was then infused with a phenolic resin (Durite SC-1008, Momentive Specialty Chemicals Inc.) at $40^{\circ} \mathrm{C}$ under vacuum for $\sim 24$ hours, forming the CNT A-PNC precursors. The polymer precursors were then cured for 6 hours at $80^{\circ} \mathrm{C}$. To turn the cured CNT A$\mathrm{PNCs}$ into A-CMNCs, samples were heat treated as follows in a He environment: $400^{\circ} \mathrm{C}$ for 30 minutes, $600^{\circ} \mathrm{C}$ for 30 minutes, $750^{\circ} \mathrm{C}$ for $30 \mathrm{~min}$. These temperatures are very close to the previously reported temperatures of maximum reaction rate for a neat phenolic resin undergoing pyrolysis ${ }^{46}$. CNT A-CMNCs were then trimmed and polished, ensuring that the regions of excess $\mathrm{PyC}$ (both above and below) surrounding the original CNT forest were removed. See Fig. 2 for HRSEM micrographs of a $\sim 1$ vol. \% CNT A-CMNC illustrating the CNT alignment (Fig. 2b) and PyC matrix porosity (Fig. 2c).

The second polymer infusion was performed by first placing the CNT A-CMNCs into hollow cylindrical plastic molds, ensuring that the primary axis of the CNTs in the A-CMNCs was orthogonal to the plane of the mold. Diluted phenolic resin (in acetone), which had a dilution factor $\left(F_{\text {dilut }}\right) \simeq 60 \%$ by mass, was then added on top of the CNT A-CMNCs. $F_{\text {dilut }}$ has a significant effect on the final CNT A-CMNC density and porosity, and is discussed further in the next Section (see Eq. 6). Polymer reinfusion took place at room temperature and pressure for $\sim 72$ hours. Reinfused CNT A-CMNCs were then cured for 30 minutes at $80^{\circ} \mathrm{C}$, and subsequently pyrolyzed using the previously described conditions $\left(400^{\circ} \mathrm{C}\right.$ (30 minutes) $\rightarrow 600^{\circ} \mathrm{C}(30$ minutes $) \rightarrow 750^{\circ} \mathrm{C}(30$ minutes)). Finally, the re-infused CNT A-CMNCs were trimmed and polished, ensuring that the regions of excess phenolic resin surrounding the original CNT forest were removed.

To evaluate the apparent matrix porosity, $\phi_{m, i}^{e x p}$, in the $\sim 1$ vol. \% CNT A-CMNCs, the experimentally determined density, $\rho_{c, i}^{\text {exp }}$, was approximated by first drying the samples in a He environment, and measuring their mass, $M_{i}^{e x p}$, on a Mettler AE100 Analytical Balance, and volume after the first pyrolyzation, $V_{1}^{\exp }$. $\phi_{m, i}^{\exp }$ was then estimated using the following relationship of $\rho_{c n t}$ and $\phi_{o}$ :

$$
\phi_{m, i}^{e x p}=1-\left(\frac{M_{i}^{e x p}-\rho_{c n t} V_{1}^{e x p}\left(\frac{1-\phi_{o}}{1+\Delta V_{p, 1}^{e x p}}\right)}{\left(1-\left(\frac{1-\phi_{o}}{1+\Delta V_{p, 1}^{e x p}}\right)\right) V_{1}^{e x p} \rho_{g}}\right)
$$




\section{B. High Resolution Scanning Electron Microscopy}

To characterize the surface morphology of CNT ACMNCs, SEM analysis was performed using a JEOL 6700 cold field-emission gun scanning electron microscope using secondary electron imaging at an accelerating voltage ranging from 1.0 (for CNT forests) ${ }^{40}$ to $3.0 \mathrm{kV}$ (for CNT A-CMNCs) ${ }^{23,24}$ and a working distance of either $3.0 \mathrm{~mm}$ (Fig. 2a and Fig. 2b) or $8.0 \mathrm{~mm}$ (Fig. 2c). To examine whether the pyrolysis step affects the alignment of the CNTs in the A-CMNCs, the cross-sectional fracture surfaces of $\mathrm{a} \sim 1$ vol. \% as-grown CNT forest (Fig. 2a) and A-CMNC (Fig. 2b and Fig. 2c), prepared by cleaving parallel to the CNT alignment direction, were imaged and compared. Fig. 2b demonstrates that the CNT A-CMNCs exhibit good matrix filling while preserving

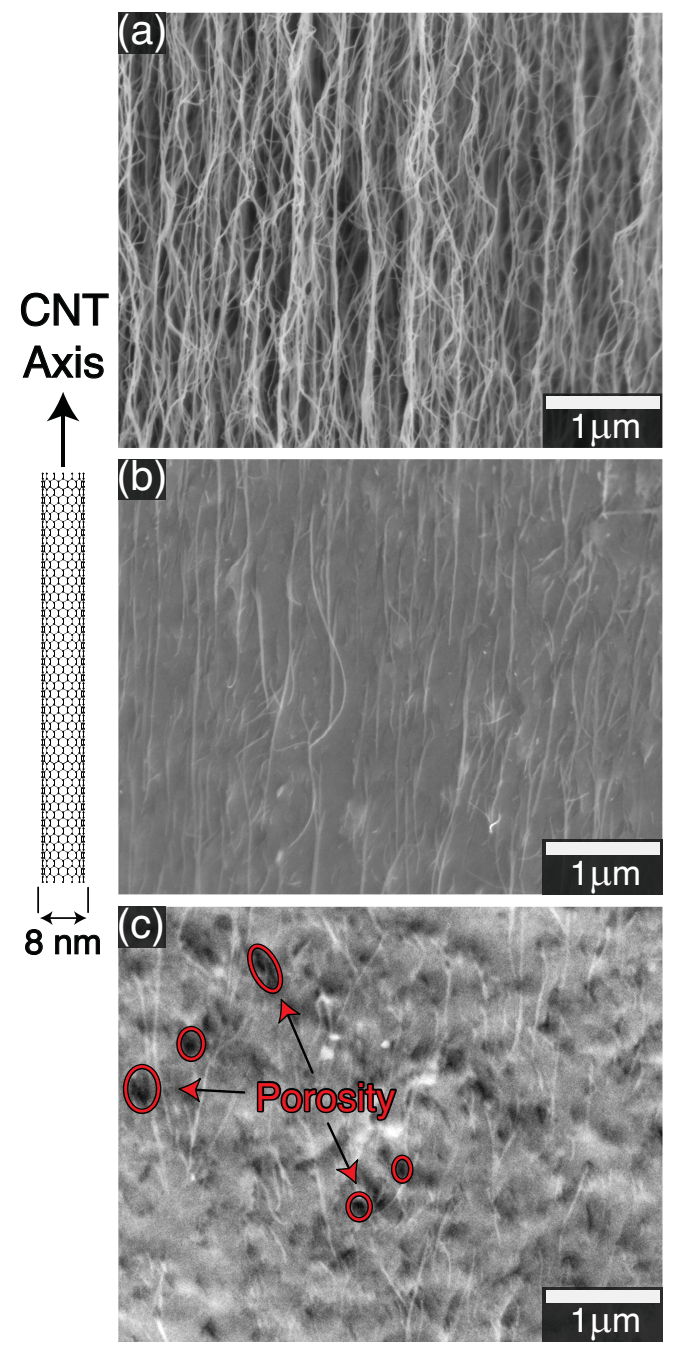

FIG. 2. HRSEM micrographs of a fracture surface of an asgrown ( $\sim 1$ vol. \%) MWCNT forest before composite fabrication (a), and after infusion and pyrolyzation (b and $\mathrm{c}$ ). The underlying CNTs can be clearly seen in (b), whereas (c) better shows the surface topology and illustrates the high $\mathrm{PyC}$ matrix porosity $(\sim 60 \%)$ of the CNT A-CMNC. the CNT alignment over a characteristic length scale of $\sim 1 \mu \mathrm{m}$. To show the high $(\sim 60 \%)$ matrix porosity in the CNT A-CMNCs, the low angle secondary electron imaging mode was used, which clearly illustrates that a significant amount of pores form during the pyrolysis process (see Fig. 2c).

\section{RESULTS AND DISCUSSION}

To quantify the effect of pyrolysis on the porosity of the CNT A-CMNCs, the experimentally determined apparent densities of the CNT A-PNCs and A-CMNCs were used to calculate $\Delta M_{p}$ and $\Delta V_{p}$ (see Table I). As illustrated in Table I, the $\sim 1$ vol. \% CNT A-CMNCs have an apparent density of $0.888 \pm 0.115 \mathrm{~g} / \mathrm{cm}^{3}$ and a calculated PyC matrix porosity of $\simeq 61.0 \pm 5.1 \%$ (evaluated using Eq. 5 and the determined $\Delta M_{p}$ and $\Delta V_{p}$ values from Table I).

The reinfusion results (see Table I) indicate that while an increase of apparent density through reinfusion with a diluted phenolic resin is feasible, it may not be the most practical way to obtain very low $\mathrm{PyC}$ matrix porosities. As shown in Table I, $\Delta M_{p, 2}^{e x p}$ is much lower than $\Delta M_{p, 1}^{e x p}$, and the following relationship between $\Delta M_{p, 1}^{\exp }, \Delta M_{p, 2}^{e x p}$, and $\xi_{2}$ is proposed:

$$
\begin{gathered}
\Delta M_{p, 2}^{e x p}=-F_{\text {dilut }} F_{\text {inf }}\left(1+\Delta M_{p, 1}^{\text {exp }}\right) \\
\xi_{2}=F_{\text {dilut }} F_{\text {inf }}\left(1+\Delta M_{p, 2}^{e x p}\right)\left(\frac{\rho_{p n c, 1}}{\rho_{g}}\right)
\end{gathered}
$$

Where $F_{\text {inf }}$ is defined as the infusion factor, which takes into account the fraction of pores that was actually infused by the diluted phenolic resin. Solving Eq. 6 for $F_{\text {inf }}$ yields $F_{\text {inf }} \sim 40 \%$, illustrating that not all of the pores were infiltrated using this method. To evaluate the maximum attainable $\rho_{c, i}$, and the number of infusions necessary to achieve it, these results were used with Eq. 4 at a range of $\psi$ values, where $\psi$ represents the fraction of pores that remains accessible after each phenolic infusion and pyrolyzation.

Since the value of $\psi$ has a very strong influence on the model predictions, a physical upper bound for $\psi\left(\psi_{\max }\right)$ was first determined. To do so, $\rho_{c, i}$ (Eq. 4) was evaluated

TABLE I. Experimentally determined A-CMNC densities $\left(\rho_{c, i}^{e x p}\right)$, calculated mass $\left(\Delta M_{p, i}^{e x p}\right)$ and volume change $\left(\Delta V_{p, i}^{e x p}\right)$ coefficients due to pyrolysis, and PyC matrix porosities $\left(\phi_{m, i}^{e x p}\right)$ evaluated using Eq. 5 for $\sim 1$ vol. \% CNT A-CMNCs fabricated using single and double polymer infusion $(i)$ and pyrolyzation.

\begin{tabular}{ccccc}
\hline \hline$i$ & $\rho_{c, i}^{\text {exp }}\left(\mathrm{g} / \mathrm{cm}^{3}\right)$ & $\Delta M_{p, i}^{\text {exp }}(\%)$ & $\Delta V_{p, i}^{\text {exp }}(\%)$ & $\phi_{m, i}^{\text {exp }}(\%)$ \\
\hline 1 & $0.888 \pm 0.115$ & $-46.7 \pm 1.1$ & $-43.1 \pm 6.6$ & $61.0 \pm 5.2$ \\
2 & $1.015 \pm 0.110$ & $-13.0 \pm 3.0$ & 0 & $54.8 \pm 4.4$ \\
\hline \hline
\end{tabular}



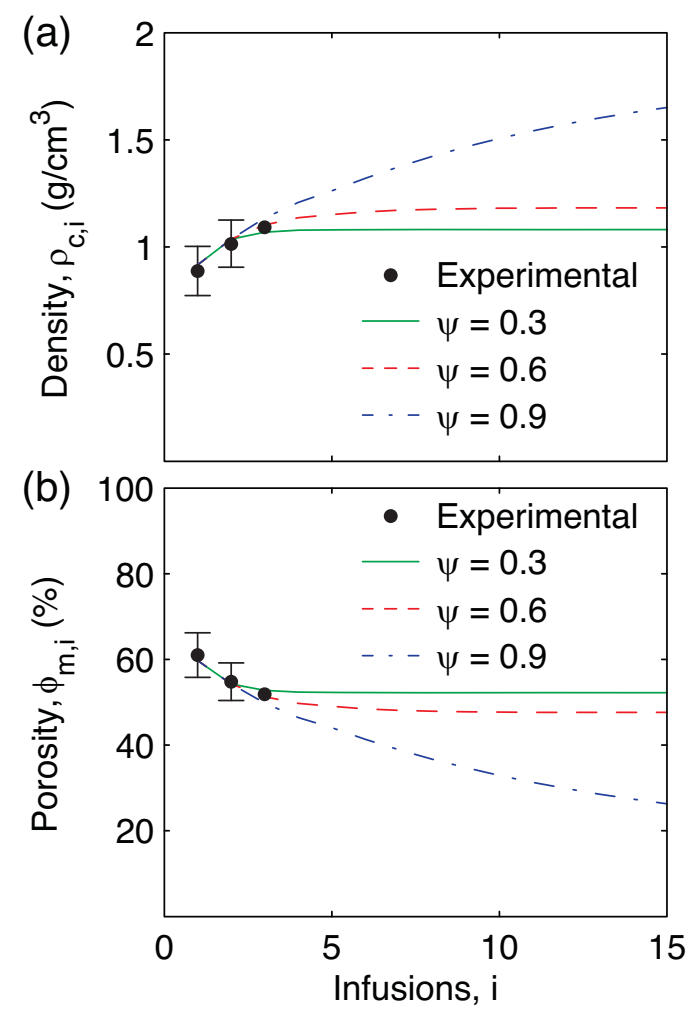

FIG. 3. Plot of the experimentally determined and model predicted A-CMNC density (a) and matrix porosity (b) as a function of number of polymer infusions $(i)$ and the fraction of pores $(\psi)$ that remains accessible after each polymer infusion and pyrolyzation. The plots illustrate that successive polymer infusions lead to significantly diminished returns at $i \geq 4$, where the $\sim 1$ vol. \% CNT A-CMNCs will have densities of $\simeq 1.1-1.2 \mathrm{~g} / \mathrm{cm}^{3}$, and $\mathrm{PyC}$ matrix porosities of $\simeq 46-52 \%$.

numerically for the case of zero matrix porosity (perfect filling), yielding $\psi_{\max } \simeq 93 \%$. See Section S3 in the Supplementary Materials for details. A preliminary study on the impact of a third polymer infusion and pyrolyzation (the second infusion with a diluted resin) on $\rho_{c, i}$ gives an estimated value of $\rho_{c, 3} \simeq 1.09 \mathrm{~g} / \mathrm{cm}^{3}(\rightarrow \psi \simeq 50 \%)^{24}$, meaning that the range of $\psi$ that will likely be phenomenologically observed is $30 \% \leq \psi \leq 90 \%$. See Fig. 3 for plots of the model predicted $\rho_{c, i}$ (Fig. 3a) and $\phi_{m, i}$ (Fig. 3b), and Table S2 in the Supplementary Materials for a list of the model predicted $\rho_{c, i}$ for $30 \% \leq \psi \leq 90 \%$. As illustrated in Fig. 3 (and Table S2 in the Supplementary Materials), regardless of the value of $\psi$, the model predicts that $\rho_{c, i}$ will not exceed $\sim 1.2 \mathrm{~g} / \mathrm{cm}^{3}$ $\left(\rightarrow \phi_{m, i} \simeq 46.4 \%\right)$ at $i=4$, where successive polymer infusions lead to significantly diminished returns for the majority of $\psi$ values (especially for $\psi \lesssim 80 \%$ ). Also, for the $\psi=90 \%$ case, in order to get $\phi_{m, i}<20 \%$, a value of $i>25$ is necessary, meaning that such a low value of $\phi_{m}$ is likely both physically and practically unattainable for A-CMNCs produced using wet infusion with a diluted $\left(F_{\text {dilut }} \simeq 60 \%\right.$ by mass $)$ phenolic resin. A possible way to achieve $\phi_{m, i}<20 \%$ at a reasonable value of $i(i \lesssim 10)$ is to apply pressure during the pyrolysis stage ${ }^{28}$, which will make $\Delta V_{p, 1} \not \equiv \Delta V_{p}$ (the simplification in Eq 3 is no longer true), but the compressive stress will probably alter the morphology of the CNTs in the matrix ${ }^{47,48}$, thereby impacting the physical properties of the CNT A-CMNCs. Therefore, in order to synthesize A-CMNCs with very low porosities $\left(\phi_{m} \lesssim 20 \%\right)$ without changing the A-CMNC morphology, a gas phase infusion method, such as carbon vapor infiltration $(\mathrm{CVI})^{27,49-51}$, would most likely be necessary. Another method that could yield very low porosity A-CMNCs is the use of successive infusions with a diluted phenolic resins, which could be designed to form approximately conformal coatings of polymer, and subsequently $\mathrm{PyC}$ (after pyrolysis). However, a drawback of such a synthesis method is the need for critical point drying $(\mathrm{CPD})^{52,53}$ to dry the samples before pyrolyzation, which is more difficult to use in an industrial scale. Future work should explore both methods, and determine which yields the CNT A-CMNCs with minimal porosities, and optimal combination of physical properties.

\section{CONCLUSIONS}

In summary, a model that can predict the attainable density and matrix porosity of aligned nanofiber architectures as a result of multiple wet polymer infusions and pyrolyzations is derived, and applied to an exemplary system of aligned nanofiber carbon matrix nanocomposites (A-CMNCs) comprised of $\sim 1$ vol. \%, $\sim 8 \mathrm{~nm}$ outer diameter, aligned CNTs. The experimental results illustrate that a second polymer infusion and pyrolyzation can increase the CNT A-CMNC density by 14\% (from $\simeq 0.888 \mathrm{~g} / \mathrm{cm}^{3}$ to $\simeq 1.015 \mathrm{~g} / \mathrm{cm}^{3}$ ) leading to a $\sim 6 \%$ reduction in the porosity of the carbon matrix (from $\simeq 61.0 \%$ to $\simeq 54.8 \%)$. Since the infusion factor had a computed value of $40 \%$, and because 10 to $70 \%$ of the remaining pores will likely become inaccessible after each polymer infusion, the model predicts significantly diminished returns will be observed after the fourth polymer infusion, where the density of the $\sim 1$ vol. \% CNT A$\mathrm{CMNC}$ will be $\simeq 1.1-1.2 \mathrm{~g} / \mathrm{cm}^{3}$ (porosity $\simeq 46-52 \%$ ). This corresponds to a $\sim 24-35 \%$ increase in density $(\sim 9-15 \%$ reduction in matrix porosity) when compared to the single polymer infusion and pyrolyzation results. These modeling results highlight that continued infusion with a diluted phenolic resin will likely never result in a porosity that is $<20 \%$, meaning that future work should explore alternative infusion methods, such as carbon vapor infiltration, and layer by layer assembly via critical point drying of thin conformal films of liquid precursor. Because high nanofiber volume fraction architectures generally have the most promising properties, but were not studied here, future work should also apply this model to A-CMNCs produced using higher CNT volume fractions $(\gtrsim 10 \text { vol. \% })^{18}$. Once gas phase infusion and layer by layer assembly methods are explored, and data for high volume fraction architectures is avail- 
able, this model could be used to accurately predict the number and type of processing steps necessary for fabrication of more general architectures of nanofibers, including nanowires, for a variety of high value applications.

\section{ACKNOWLEDGEMENTS}

I.Y.S. received partial support from the National Science Foundation under Grant No. CMMI-1130437. The authors thank Samuel T. Buschhorn and Stephen A. Steiner III for helpful discussions, and the members of necstlab for technical support and advice. This work made use of the core facilities at the Institute for Soldier Nanotechnologies at MIT, supported in part by the U.S. Army Research Office under contract W911NF-07D-0004.

\section{Appendix A: Supplementary data}

Supplementary data associated with this article can be found, in the online version, at http://dx.doi.org/10.1016/j.carbon.2013.12.001.
* iys@mit.edu.

† wardle@mit.edu.

1 W. U. Huynh, J. J. Dittmer, and A. P. Alivisatos, Science 295, 2425 (2002).

2 P. Joshi, L. Zhang, D. Davoux, Z. Zhu, D. Galipeau, H. Fong, and Q. Qiao, Energy Environ. Sci. 3, 1507 (2010).

3 W. Gaynor, G. F. Burkhard, M. D. McGehee, and P. Peumans, Adv. Mater. 23, 2905 (2011).

${ }^{4}$ C.-H. Chung, T.-B. Song, B. Bob, R. Zhu, H.-S. Duan, and Y. Yang, Adv. Mater. 24, 5499 (2012).

5 V. H. Luan, H. N. Tien, T. V. Cuong, B.-S. Kong, J. S. Chung, E. J. Kim, and S. H. Hur, J. Mater. Chem. 22, 8649 (2012).

${ }^{6}$ P. Poudel and Q. Qiao, Nanoscale 4, 2826 (2012).

7 T. Nagai, N. Aoki, Y. Ochiai, and K. Hoshino, ACS Appl. Mater. Interfaces 3, 2341 (2011).

${ }^{8}$ H. Huang, B. Liang, Z. Liu, X. Wang, D. Chen, and G. Shen, J. Mater. Chem. 22, 13428 (2012).

9 Z. Chen, V. Augustyn, X. Jia, Q. Xiao, B. Dunn, and Y. Lu, ACS Nano 6, 4319 (2012).

10 J. H. Jung, C.-Y. Chen, B. K. Yun, N. Lee, Y. Zhou, W. Jo, L.-J. Chou, and Z. Lin, Nanotechnology 23, 375401 (2012).

11 A. Vlad, A. L. M. Reddy, A. Ajayan, N. Singh, J.-F. Gohy, S. Melinte, and P. M. Ajayan, Proc. Natl. Acad. Sci. U. S. A. 109, 15168 (2012).

12 M. Bozlar, D. He, J. Bai, Y. Chalopin, N. Mingo, and S. Volz, Adv. Mater. 22, 1654 (2010).

13 S. Shen, A. Henry, J. Tong, R. Zheng, and G. Chen, Nat. Nanotechnol. 5, 251 (2010).

14 L. Zhang, G. Zhang, C. Liu, and S. Fan, Nano Lett. 12, 4848 (2012).

15 S. Vaddiraju, H. Cebeci, K. K. Gleason, and B. L. Wardle, ACS Appl. Mater. Interfaces 1, 2565 (2009).

16 A. M. Marconnet, N. Yamamoto, M. A. Panzer, B. L. Wardle, and K. E. Goodson, ACS Nano 5, 4818 (2011).

17 E. J. Garcia, B. L. Wardle, A. J. Hart, and N. Yamamoto, Compos. Sci. Technol. 68, 2034 (2008).

18 B. L. Wardle, D. S. Saito, E. J. García, A. J. Hart, R. Guzmán de Villoria, and E. A. Verploegen, Adv. Mater. 20, 2707 (2008).

19 M. K. Shin, J. Oh, M. Lima, M. E. Kozlov, S. J. Kim, and R. H. Baughman, Adv. Mater. 22, 2663 (2010).

20 A. T. Sepúlveda, R. Guzmán de Villoria, J. C. Viana, A. J. Pontes, B. L. Wardle, and L. A. Rocha, Nanoscale 5, 4847 (2013).
21 L. Liu, W. Ma, and Z. Zhang, Small 7, 1504 (2011).

${ }^{22}$ L. Ci, J. Suhr, V. Pushparaj, X. Zhang, and P. M. Ajayan, Nano Lett. 8, 2762 (2008).

23 I. Y. Stein, H. M. Vincent, S. A. Steiner, E. Colombini, and B. L. Wardle, in 54th AIAA Structures, Structural Dynamics, and Materials (SDM) Conference (Boston, MA, 2013).

${ }^{24}$ I. Y. Stein, Synthesis and Characterization of NextGeneration Multifunctional Material Architectures: Aligned Carbon Nanotube Carbon Matrix Nanocomposites, Master's thesis, Massachusetts Institute of Technology (2013).

25 L. An, W. Xu, S. Rajagopalan, C. Wang, H. Wang, Y. Fan, L. Zhang, D. Jiang, J. Kapat, L. Chow, B. Guo, J. Liang, and R. Vaidyanathan, Adv. Mater. 16, 2036 (2004).

26 S. Sarkar, J. Zou, J. Liu, C. Xu, L. An, and L. Zhai, ACS Appl. Mater. Interfaces 2, 1150 (2010).

27 X. Li, K. Li, H. Li, J. Wei, and C. Wang, Carbon 45, 1662 (2007).

28 D. Bansal, S. Pillay, and U. Vaidya, Carbon 55, 233 (2013).

29 J. P. Lu, Phys. Rev. Lett. 79, 1297 (1997).

30 E. W. Wong, P. E. Sheehan, and C. M. Lieber, Science 277, 1971 (1997).

31 D. A. Walters, L. M. Ericson, M. J. Casavant, J. Liu, D. T. Colbert, K. A. Smith, and R. E. Smalley, Appl. Phys. Lett. 74, 3803 (1999).

32 M.-F. Yu, B. S. Files, S. Arepalli, and R. S. Ruoff, Phys. Rev. Lett. 84, 5552 (2000).

33 M. F. L. De Volder, S. H. Tawfick, R. H. Baughman, and A. J. Hart, Science 339, 535 (2013).

34 S. Frank, P. Poncharal, Z. L. Wang, and W. A. de Heer, Science 280, 1744 (1998).

35 P. Kim, L. Shi, A. Majumdar, and P. L. McEuen, Phys. Rev. Lett. 87, 215502 (2001).

36 M. Kociak, A. Y. Kasumov, S. Guron, B. Reulet, I. I. Khodos, Y. B. Gorbatov, V. T. Volkov, L. Vaccarini, and H. Bouchiat, Phys. Rev. Lett. 86, 2416 (2001).

37 W. Liang, M. Bockrath, D. Bozovic, J. H. Hafner, M. Tinkham, and H. Park, Nature 411, 665 (2001).

38 Z. K. Tang, L. Zhang, N. Wang, X. X. Zhang, G. H. Wen, G. D. Li, J. N. Wang, C. T. Chan, and P. Sheng, Science 292, 2462 (2001).

39 R. H. Baughman, A. A. Zakhidov, and W. A. de Heer, Science 297, 787 (2002). 
${ }^{40}$ I. Y. Stein and B. L. Wardle, Phys. Chem. Chem. Phys. 15, 4033 (2013).

41 A. J. Hart and A. H. Slocum, J. Phys. Chem. B 110, 8250 (2006).

42 C. Laurent, E. Flahaut, and A. Peigney, Carbon 48, 2994 (2010).

43 S. S. Wicks, R. Guzman de Villoria, and B. L. Wardle, Compos. Sci. Technol. 70, 20 (2010).

${ }^{44}$ H. Qian, E. S. Greenhalgh, M. S. P. Shaffer, and A. Bismarck, J. Mater. Chem. 20, 4751 (2010).

45 R. R. Mitchell, N. Yamamoto, H. Cebeci, B. L. Wardle, and C. V. Thompson, Compos. Sci. Technol. 74, 205 (2013).

46 K. A. Trick and T. E. Saliba, Carbon 33, 1509 (1995).
47 C. Cao, A. Reiner, C. Chung, S.-H. Chang, I. Kao, R. V. Kukta, and C. S. Korach, Carbon 49, 3190 (2011).

48 A. Qiu, D. Bahr, A. Zbib, A. Bellou, S. Mesarovic, D. McClain, W. Hudson, J. Jiao, D. Kiener, and M. Cordill, Carbon 49, 1430 (2011).

49 Q.-M. Gong, Z. Li, X.-D. Bai, D. Li, Y. Zhao, and J. Liang, Mater. Sci. Eng., A 384, 209 (2004).

50 Q.-M. Gong, Z. Li, D. Li, X.-D. Bai, and J. Liang, Solid State Commun. 131, 399 (2004).

51 J. Li, R. Luo, and Y. Yan, Carbon 49, 242 (2011).

52 M. Bryning, D. Milkie, M. Islam, L. Hough, J. Kikkawa, and A. Yodh, Adv. Mater. 19, 661 (2007).

${ }^{53}$ K. H. Kim, Y. Oh, and M. F. Islam, Nat. Nanotechnol. 7, 562 (2012). 


\title{
Supplementary Information: Morphology and Processing of Aligned Carbon Nanotube Carbon Matrix Nanocomposites
}

\author{
Itai Y. Stein* \\ Department of Mechanical Engineering, \\ Massachusetts Institute of Technology, \\ 77 Massachusetts Ave, Cambridge, MA 02139, USA. \\ Brian L. Wardle ${ }^{\dagger}$ \\ Department of Aeronautics and Astronautics, \\ Massachusetts Institute of Technology, \\ 77 Massachusetts Ave, Cambridge, MA 02139, USA.
}

\footnotetext{
* iys@mit.edu.

† wardle@mit.edu.
} 


\section{S1. EXPANDED FORM OF INTERMEDIATE REINFUSION MODEL EQUA- TIONS}

The density of the $\mathrm{PyC}$ matrix after the first polymer infusion and pyrolyzation, $\rho_{m, 1}$, can be defined as follows (expanded from Eq. 1 in the main text):

$$
\rho_{m, 1}=\rho_{g}\left(1-\frac{1-\phi_{o}}{1+\Delta V_{p, 1}}\right)\left(1-\frac{\phi_{p n c, 1}\left(1+\Delta M_{p, 1}\right)}{1+\Delta V_{p, 1}}\right)
$$

To calculate the density for a non-constant rate of change after $i$ infusions and pyrolyzation,

$\rho_{m, i}$, the change in mass, $\Delta M_{p, i}$, and volume, $\Delta V_{p, i}$, of the system needs be taken into account, leading to the following summation:

$$
\rho_{m, i}=\frac{M_{i}-\sum_{j=1}^{i-1}\left(M_{j}\right)\left(1+\Delta M_{p, j}\right)}{V_{i}-\sum_{j=1}^{i-1} V_{j}\left(1-\frac{\rho_{p n c, j}}{\rho_{g}}\left(1+\Delta M_{p, j}\right)\right)\left(1+\Delta V_{p, j}\right)}
$$

Eq. S2 can be extended to the following general form:

$$
\begin{array}{r}
\rho_{m, i}=\rho_{g}\left(1-\frac{1-\phi_{o}}{1+\Delta V_{p, 1}+\Delta V_{p, 2}+\ldots}\right)\left(1-\left(\frac{\rho_{p n c, 1}\left(1+\Delta M_{p, 1}\right)}{\rho_{g}\left(1+\Delta V_{p, 1}\right)}\right)\right. \\
-\left(\left(\frac{\rho_{p n c, 2}\left(1+\Delta M_{p, 2}\right)}{\rho_{g}\left(1+\Delta V_{p, 2}\right)}\right)\left(\frac{\rho_{p n c, 1}\left(1+\Delta M_{p, 1}\right)}{\rho_{g}\left(1+\Delta V_{p, 1}\right)}\right)\right)-\left(\frac{\rho_{p n c, 3}\left(1+\Delta M_{p, 3}\right)}{\rho_{g}\left(1+\Delta V_{p, 3}\right)}\right) \\
\left.\left(\left(1-\frac{\rho_{p n c, 2}\left(1+\Delta M_{p, 2}\right)}{\rho_{g}\left(1+\Delta V_{p, 2}\right)}\right)\left(\frac{\rho_{p n c, 1}-\left(1+\Delta M_{p, 1}\right)}{\rho_{g}\left(1+\Delta V_{p, 1}\right)}\right)\right)-\ldots\right)
\end{array}
$$

See Eq. 3 in the main text for the condensed form. 


\section{S2. MULTIWALLED CNT INTRINSIC DENSITY CALCULATION}

(a)

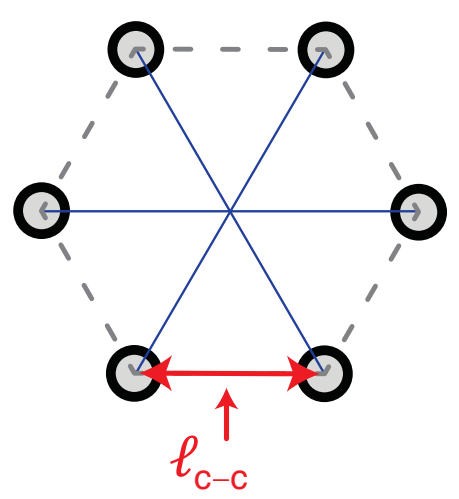

(b)

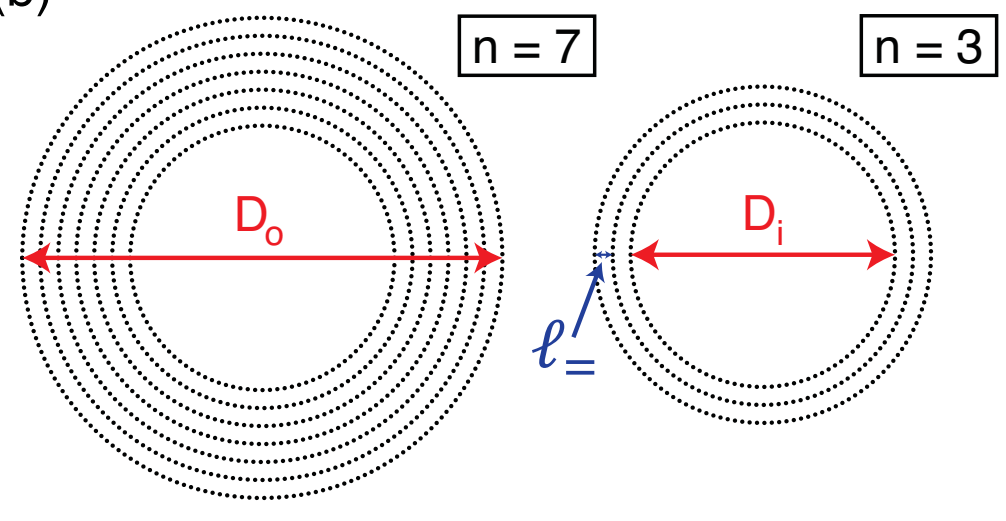

FIG. S1. Illustration of the geometry used to compute the theoretical density of graphene (a), and the cross-sectional geometry of the MWCNTs (b) ranging from 3 to 7 walls $(3 \leq n \leq 7)$. The inter-layer spacing, $\ell_{=}$, the $\mathrm{C}-\mathrm{C}$ bond length, $\ell_{c-c}$, and the MWCNT inner, $D_{i}$, and outer, $D_{o}$, diameters are indicated.

To evaluate the theoretical intrinsic density of a CNT, its structure and bonding must be considered. For an MWCNT, which is composed of layers of rolled up graphene, the theoretical density of a single graphene sheet, $\rho_{g}$, needs to first be evaluated. To predict the density of graphene, the density of the repeat unit (benzene ring) must be evaluated (see Fig. S1a for illustration). Using the mass of carbon atoms, $M_{C}$, their van der Waals radius, $r_{v d W}$, and the carbon-carbon bond length, $\ell_{c-c}$, the following expression can be derived for the density of a single graphene sheet, $\rho_{g}$ :

$$
\rho_{g}=\frac{2 \sqrt{3} M_{C}}{9 \ell_{c-c}^{2} r_{v d W}}
$$

Using $r_{v d W}=\frac{1}{2} \ell_{=} \simeq 1.705 \AA$ and $\ell_{c-c}=1.415 \AA$, the predicted $\rho_{g}$ is $\simeq 2.25 \mathrm{~g} / \mathrm{cm}^{3}$, which is the same as single crystal graphite.

To find the density of a MWCNT with $n$ number of walls, the effective volume of graphene needs to be evaluated. Using a reasonable inter-layer spacing value for MWCNTs, $\ell=(\approx$ $3.41 \AA$ ), and the MWCNT inner, $D_{i}$, and outer, $D_{o}$, diameters, the effective volume of graphene as a function of CNT length and number of walls, $V_{g, n}^{\prime}$, and the density of a MWCNT with $n$ walls, $\rho_{c n t, n}$, can be evaluated (see Fig. S1b for an illustration of the 
MWCNT cross-sectional geometry):

$$
\begin{gathered}
V_{g, n}^{\prime}=\pi \ell_{=}\left(\sum_{j=1}^{n}\left(D_{i}+2 \ell_{=}(j-1)\right)\right) \\
\rho_{c n t, n}=4 \rho_{g} \ell_{=}\left(\frac{\sum_{j=1}^{n}\left(D_{i}+2 \ell_{=}(j-1)\right)}{D_{o}^{2}}\right)
\end{gathered}
$$

See Fig. S2a for a plot of the intrinsic MWCNT densities, computed using Eq. S6, as a function of $n$ for $3 \leq n \leq 7$. Since the MWCNT forests used in this study are composed of a distribution of $3-7$ walled MWCNTs with $D_{o}=\left(D_{i}+2 \ell_{=}(n-1)\right)^{2}$, the average MWCNT intrinsic density, $\rho_{c n t}$, must also take into account the normalized population of each $n$ walled MWCNT, $p_{n}$, and needs to be computed as follows:

$$
\begin{array}{r}
\rho_{\text {cnt }}=4 \rho_{g} \ell_{l}=\left(\sum_{k=3}^{7} \frac{p_{k}}{\left(D_{i}+2 \ell_{=}(k-1)\right)^{2}}\right. \\
\left.\left(\sum_{j=1}^{k}\left(D_{i}+2 \ell_{=}(j-1)\right)\right)\right)
\end{array}
$$

(a)

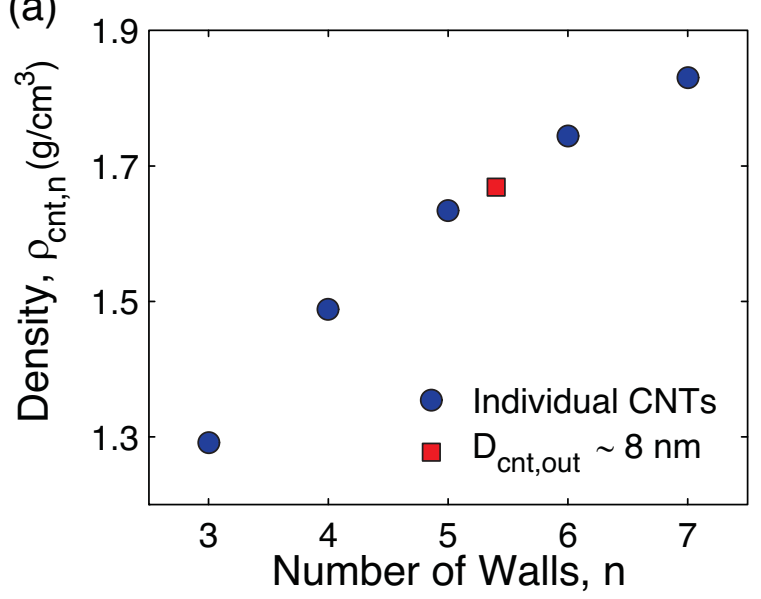

(b) Average Density, $\rho_{\text {cnt }}=1.66873 \mathrm{~g} / \mathrm{cm}^{3}$

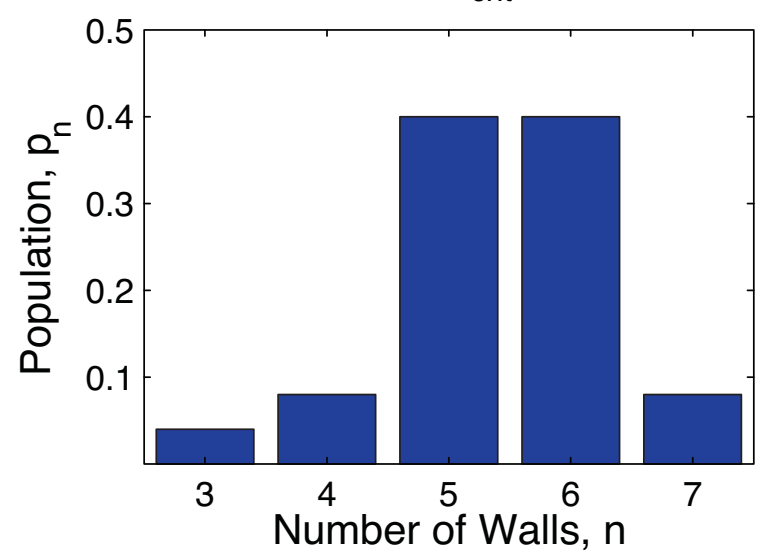

FIG. S2. Plot of the computed intrinsic MWCNT densities, $\rho_{c n t, n}$, (from Eq. S7) as a function of number of walls (a), defined as $n$, for $3 \leq n \leq 7$, and histogram of the numerically determined average population of MWCNTs $\left(p_{n}\right)$ in a $\sim 8 \mathrm{~nm}$ average outer diameter CNT forest. The average intrinsic CNT density in the forest, $\rho_{c n t}$, for the evaluated population (b) is $\rho_{c n t} \simeq 1.7 \mathrm{~g} / \mathrm{cm}^{3}$. 
(a)

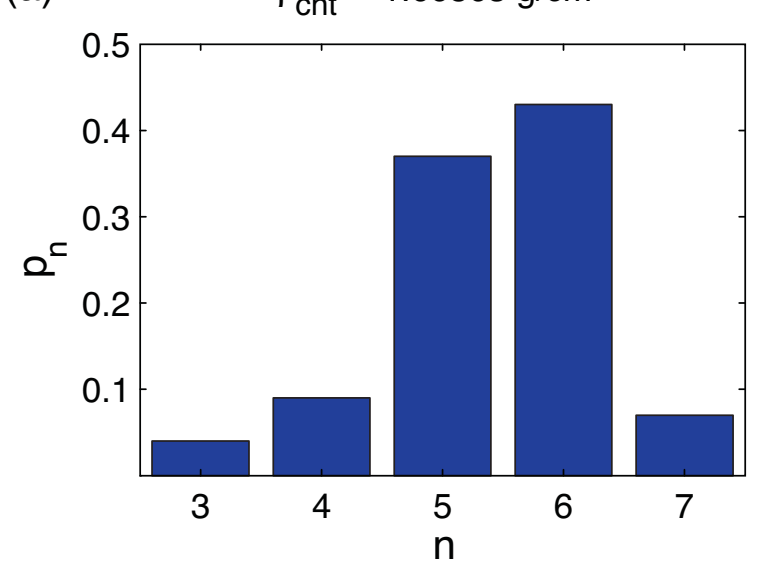

(b)

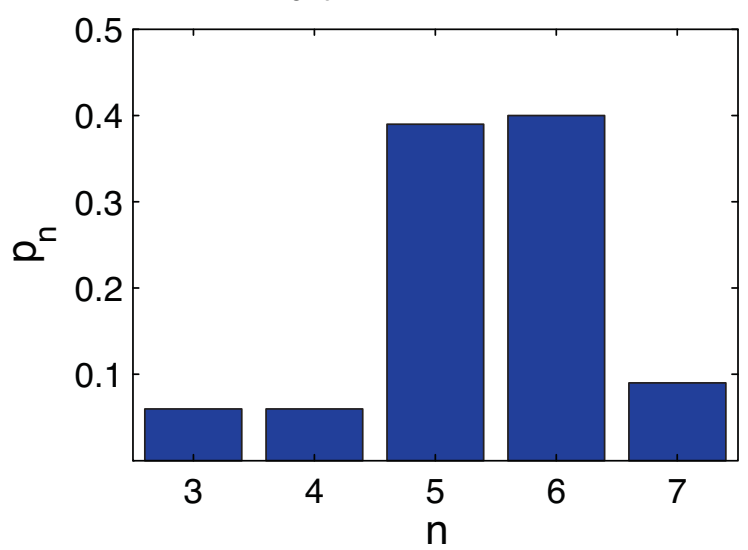

FIG. S3. Plots of two additional numerical solutions (solved using Eq. S7) for the normalized population profile of MWCNTs $\left(p_{n}\right)$ as a function of their number of walls $(n)$ in a $\sim 8 \mathrm{~nm}$ average outer diameter CNT forest. The plots illustrate that the average intrinsic density of the CNTs $\left(\rho_{\text {cnt }}\right)$ used in this study is $\sim 1.7 \mathrm{~g} / \mathrm{cm}^{3}$.

A previous high resolution transmission electron microscopy (HRTEM) study found that the average values of $D_{i}$ and $D_{o}$ for our CNTs are $D_{i} \approx 5 \mathrm{~nm}$ and $D_{o} \approx 8 \mathrm{~nm}$, meaning that the population profile can be evaluated numerically, thereby yielding a family of solutions that includes the following: $p_{3} \approx 0.04 ; p_{4} \approx 0.08 ; p_{5} \approx 0.40 ; p_{6} \approx 0.40 ; p_{7} \approx 0.08$. A plot of these results can be found in Fig. S2b. See Fig. S3 and Table S1 for two more numerical solutions that belong to this family. Evaluating Eq. S7 using $\rho_{g}$ from Eq. S4 and the above populations yields a predicted $\rho_{\text {cnt }}$ value of $1.66873 \mathrm{~g} / \mathrm{cm}^{3} \approx 1.7 \mathrm{~g} / \mathrm{cm}^{3}$. This value of $\rho_{\text {cnt }}$ is in very good agreement with the $\rho_{\text {cnt }}$ values computed for the two other numerical solutions $\left(1.66863 \mathrm{~g} / \mathrm{cm}^{3} \approx 1.7 \mathrm{~g} / \mathrm{cm}^{3}\right.$ and $\left.1.66674 \mathrm{~g} / \mathrm{cm}^{3} \approx 1.7 \mathrm{~g} / \mathrm{cm}^{3}\right)$. These $\rho_{\text {cnt }}$ values, along with the corresponding $p_{n}$ values, can be found in Table S1.

TABLE S1. Normalized population profile of MWCNTs $\left(p_{n}\right)$ as a function of their number of walls $(n)$ from Fig. S2 and the corresponding average intrinsic CNT density $\left(\rho_{c n t}\right)$ determined numerically using Eq. 9 of the main text.

\begin{tabular}{cccccc}
\hline \hline$p_{3}$ & $p_{4}$ & $p_{5}$ & $p_{6}$ & $p_{7}$ & $\rho_{\text {cnt }}\left(\mathrm{g} / \mathrm{cm}^{3}\right)$ \\
\hline 0.04 & 0.09 & 0.37 & 0.43 & 0.07 & 1.66863 \\
0.06 & 0.06 & 0.39 & 0.40 & 0.09 & 1.66674 \\
\hline \hline
\end{tabular}


S3. NUMERICAL SOLUTION FOR UPPER BOUND ON ACCESSIBLE POROSITY

To determine the physical upper bound on fraction of pores that remains accessible after each phenolic infusion and pyrolyzation, $\psi_{\max }$, Eq. 4 from the main text (in its full form) was used to estimate the maximum of the CNT A-CMNC density, $\rho_{c, \text { max }}$, which would occur at zero matrix porosity (perfect filling):

$$
\begin{gathered}
\rho_{c, \text { max }}=\rho_{g}\left(1-\frac{1-\phi_{o}}{1+\Delta V_{p}}\right)+\rho_{c n t}\left(\frac{1-\phi_{o}}{1+\Delta V_{p}}\right) \\
\hookrightarrow \rho_{c, \text { max }} \simeq 2.24 \mathrm{~g} / \mathrm{cm}^{3}
\end{gathered}
$$

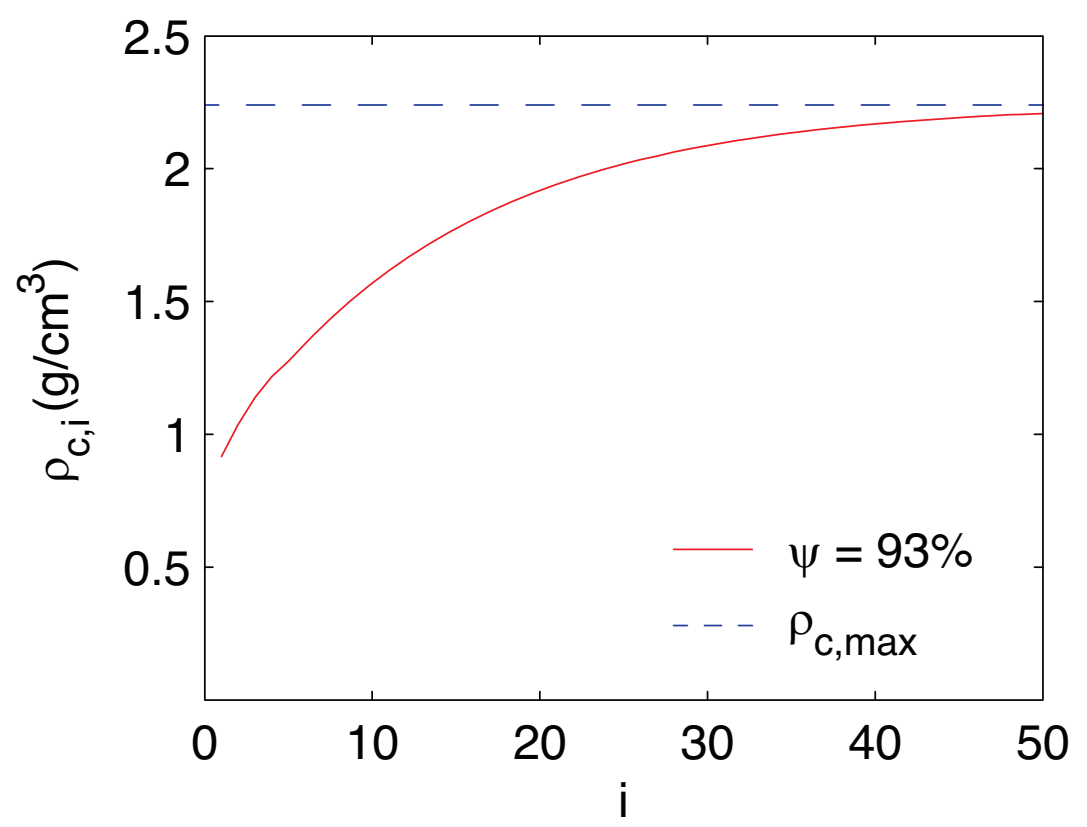

FIG. S4. Plot of the CNT A-CMNC density, $\rho_{c, i}$, as a function of polymer infusions , $i$, for $\psi=\psi_{\max } \simeq 93 \%$, showing that the model predicted $\rho_{c, i}$ approaches, but never exceeds, $\rho_{c, \max }$ from Eq. S8. 


\section{S4. MODEL PREDICTED A-CMNC DENSITY VALUES}

TABLE S2. Model predicted CNT A-CMNC density $\left(\rho_{c, i}\right)$ values as a function of number of polymer infusions $(i)$ for $30 \% \leq \psi \leq 90 \%$, as plotted in Fig. 4a in the main text.

\begin{tabular}{cccc}
\hline \hline$i$ & $\rho_{c, i}(\psi=30 \%)\left(\mathrm{g} / \mathrm{cm}^{3}\right)$ & $\rho_{c, i}(\psi=60 \%)\left(\mathrm{g} / \mathrm{cm}^{3}\right)$ & $\rho_{c, i}(\psi=90 \%)\left(\mathrm{g} / \mathrm{cm}^{3}\right)$ \\
\hline 1 & 0.9161 & 0.9161 & 0.9161 \\
2 & 1.0365 & 1.0365 & 1.0365 \\
3 & 1.0693 & 1.1022 & 1.1350 \\
4 & 1.0780 & 1.1358 & 1.2083 \\
5 & 1.0797 & 1.1515 & 1.2615 \\
6 & 1.0805 & 1.1640 & 1.3202 \\
7 & 1.0808 & 1.1716 & 1.3742 \\
8 & 1.0808 & 1.1761 & 1.4229 \\
9 & 1.0809 & 1.1789 & 1.4667 \\
10 & 1.0809 & 1.1805 & 1.5061 \\
11 & 1.0809 & 1.1815 & 1.5416 \\
12 & 1.0809 & 1.1821 & 1.5736 \\
13 & 1.0809 & 1.1824 & 1.6023 \\
14 & 1.0809 & 1.1826 & 1.6281 \\
15 & 1.0809 & 1.1828 & 1.6515 \\
\hline \hline
\end{tabular}

